ÉGYPTE

monde arabe

\section{Égypte/Monde arabe}

30-31 | 1997

Les visions de l'Occident dans le monde arabe

\title{
Jeunes Égyptiens et jeunes Marocains face à
}

l'Occident

Appropriation, attrait, répulsion

\section{Mounia Bennani-Chraïbi}

\section{OpenEdition}

\section{Journals}

Édition électronique

URL : https://journals.openedition.org/ema/1605

DOI : 10.4000/ema.1605

ISSN : 2090-7273

Éditeur

CEDEJ - Centre d'études et de documentation économiques juridiques et sociales

Édition imprimée

Date de publication : 30 septembre 1997

Pagination : 115-144

ISSN : 1110-5097

Référence électronique

Mounia Bennani-Chraïbi, « Jeunes Égyptiens et jeunes Marocains face à l'Occident », Égypte/Monde arabe [En ligne], 30-31 | 1997, mis en ligne le 08 juillet 2008, consulté le 07 juillet 2022. URL : http:// journals.openedition.org/ema/1605; DOI : https://doi.org/10.4000/ema.1605

Ce document a été généré automatiquement le 7 juillet 2022

Tous droits réservés 


\title{
Jeunes Égyptiens et jeunes Marocains face à l'Occident
}

\author{
Appropriation, attrait, répulsion
}

\section{Mounia Bennani-Chraïbi}

$1 \mathrm{Au}$ Maroc comme en Égypte, l'Occident est à la fois source de fascination et objet de diabolisation. On a, de longue date et à partir de sources dites savantes, tenté de répertorier les indicateurs de cette tradition à la fois européenne et islamique de composition de l'image de soi sur la base de la dynamique de l'altérité (Laroui, 1987). Ce n'est toutefois pas là notre propos. Relever quelques mécanismes de construction des significations autour de l'Ailleurs; observer les configurations de cet Ailleurs ou de cet "Occident"; analyser les variations de celles-ci en corrélation avec les modes d'insertion des individus et des groupes dans la ville et dans l'univers de ladite globalisation; resituer l'ensemble dans les histoires nationales respectives: telle est notre perspective. Dans quelle mesure l'ensemble de ces élaborations ou de ces bricolages épouse-t-il à la fois des dynamiques transnationales, des redéfinitions des relations sociales, politiques, et des enjeux stratégiques internes à un contexte national spécifique? Là réside l'intérêt de mener une comparaison entre deux pays arabomusulmans de la rive sud de la Méditerranée qui ont eu, au cours de la seconde moitié du $\mathrm{XX}^{\mathrm{e}}$ siècle, des stratégies contrastées de développement et de positionnement sur la scène internationale.

2 La géographie de l'Occident n'est pas la même, selon qu'elle est esquissée à partir de Casablanca ou du Caire. Si la France et, dans une moindre mesure, l'Espagne, c'est-àdire les anciennes puissances tutélaires, continuent à prédominer au Maroc, tant en ce qui concerne les échanges touristiques, migratoires, économiques, que dans le rôle de médiation ou de support privilégié que ces pays jouent au niveau de l'orientation des attentes et des remontrances, il en va tout autrement en Égypte. En termes de flux migratoires, cette République est davantage tournée vers la Péninsule arabique. Son histoire récente la lie à la Grande-Bretagne et sa politique de libéralisation (infitâh) aux États-Unis. La position qu'elle occupe sur la scène arabe, les guerres qu'elle a connues (1956 : nationalisation du canal de Suez ; 1962-1967 : guerre du Yémen ; 1967 : guerre 
des Six Jours; 1973 : guerre d'Octobre contre Israël), marquent la spécificité de sa trajectoire par rapport à la région (Vatikiotis, 1991).

Dans ce contexte, les jeunes constituent un révélateur de la construction de l'altérité. En Égypte comme au Maroc, plus des deux tiers de la population ont moins de trente ans. En raison du déclin de la mortalité juvénile après les indépendances et de la baisse du taux d'accroissement de la population à partir de 1985, le poids des jeunes n'a jamais été et ne sera jamais aussi important (Fargues, 1994, p. 163-197). L'intégration socioéconomique des moins de trente ans est le problème majeur des pays de la rive sud de la Méditerranée. Cette tranche de la population, en phase de transition, est davantage exposée à l'appel de l'Ailleurs. Elle apparaît donc comme l'acteur privilégié des transformations en cours dans la société 1 .

\section{Trajets et territoires de l'ailleurs}

4 En Égypte comme au Maroc, l'Occident chemine par des canaux humains. Le voyage à l'étranger demeure la panacée des élites et dans une certaine mesure, d'une partie des classes moyennes dotées d'un pouvoir d'achat suffisamment élevé et d'une formation au moins biculturelle. Aussi bien, le contact avec l'extérieur s'établit-il essentiellement via le tourisme et l'émigration, notamment en ce qui concerne le Maroc. L'importante communauté marocaine ${ }^{2}$ résidant en France, au Benelux, en Allemagne et plus récemment en Italie et en Espagne, maintient des liens avec son pays d'origine. En été, l'heure est aux retrouvailles; la plupart des jeunes ont un proche à l'étranger : père, frère ou soeur, membres de la famille élargie, amis d'enfance, camarades de classe, voisins ou simples connaissances.

5 L'autre grand pont est indéniablement jeté par le tourisme, l'une des principales sources en devises du Maroc et de l'Égypte. Cette présence étrangère saisonnière met les jeunes Marocains au contact de populations venues de loin. Les Européens, ressortissants des anciennes puissances protectrices en tête, représentent le gros des bataillons de touristes dans le royaume ${ }^{3}$. Des jeunes n'hésitent pas à aller à leur devant ; des relations se nouent à partir d'une demande de renseignements, sur la plage grâce au voisinage de deux serviettes ou à une partie de volley-ball : discussions, échanges d'informations et d'adresses s'ensuivent. La correspondance prend souvent le relais de ces rencontres de hasard ${ }^{4}$.

6 Au Caire, à Marrakech, à Fès, à Tanger, certains espaces privilégient plus que d'autres l'exposition à l'Occident. Les lieux touristiques constituent des univers quasiment à part. Ils rassemblent des populations qui se frottent quotidiennement à des voyageurs en quête d'exotisme. Le lieu d'affluence, symbole d'une civilisation, d'une culture, se transforme en territoire où l'autre en visite s'offre à la découverte, au jeu de séduction, à la conquête. Pour le guide, pour le vendeur de souvenirs, le loueur de chevaux et de dromadaires, il s'agit certes d'instaurer une relation marchande, mais aussi de voyager chez soi, et éventuellement de cumuler les atouts pour aller chez l'autre. Aussi bien connaissent-ils des expressions passe-partout dans plusieurs langues. Leur «look» semble parfois accréditer la thèse d'une culture mondialisée.

7 «Territoires jeunes» (Depaule, 1990, p. 153-161) et vitrines de l'Occident fusionnent par moment. Il en est ainsi, à Casablanca comme au Caire, des chaînes de fast-foods tels que le MacDonald's, le Pizza Hut, etc. Certes, ils ne sont pas exclusivement fréquentés par une population donnée: outre quelques étrangers, des personnes viennent en 
famille. Cependant, les jeunes, qui n'appartiennent pas tous aux classes supérieures, imprègnent ces lieux par leur présence et s'en imprègnent. Leur manière de se vêtir, de se retrouver en groupe, l'ambiance musicale dans laquelle ils se fondent, tout cela laisse entrevoir des modes de participation à une société de consommation transnationale. Plus généralement dans les espaces de prédilection des moins de trente ans, rythmes arabes et tubes occidentaux se succèdent et alternent. Samedi, dans le parc d'attraction de Qanâtir, dans les environs du Caire, bandes de garçons, groupes de jeunes filles en hijâb ou non, travaillant presque uniformément un look "jeune", dansent sur les cadences de la musique orientale, du Rapp ou de la Macarena. Alors qu'en Egypte, l'Ailleurs occidental semble enserré dans une territorialité, au Maroc, sa localisation est plus diffuse : Michael Jackson, les lecteurs de Coran et Um Kulthûm battent la mesure du souk, lieu de fourmillement des objets de contrefaçon et de contrebande. Ici et là dans les lieux publics marocains, s'entrechoquent les sonorités des stations locales de radiodiffusion : arabes, berbères, française, bilingue.

8 Les chaînes de télévision marocaines se distinguaient déjà par une programmation multiculturelle sur le mode bilingue et les images espagnoles arrosaient depuis des décennies le nord du pays. L'arrivée des antennes paraboliques a cependant élargi le champ de l'Ailleurs aussi bien en Égypte que dans le royaume, où ce phénomène gagne même les bidonvilles. Au Maroc, le marché de l'écrit n'échappe pas à cette variété : le français, enseigné dès la troisième année de l'école primaire publique, est demeuré l'un des véhicules privilégiés de l'enseignement jusqu'en 1983, date de l'amorce du processus d'arabisation des disciplines scientifiques ${ }^{5}$. Dans les kiosques, traductions, publications internationales arabes, françaises ou même anglo-saxonnes, côtoient les produits français et arabes de l'édition nationale. Enfin, la plupart des grands quotidiens marocains paraissent dans les deux langues.

Grâce à ces canaux, les jeunes réussissent à recueillir sur l'Occident un savoir - très précis au Maroc -, renvoyant au vécu de leurs relations à l'étranger, aux noms des firmes qui les emploient, à la localité où ils habitent, etc. Tous ces éléments font de cet univers un espace à la fois proche et lointain, favorisant de la sorte une forte éclosion de représentations obéissant à trois types de mécanismes : l'appropriation, l'attraction et la répulsion. L'altérité sous-tend les dynamiques de fascination et de diabolisation. Mais il arrive aussi que ce qui vient du dehors soit approprié tant au niveau des pratiques qu'à celui des représentations.

\section{Appropriation}

10 Le fourmillement des signes de l'Ailleurs, et plus précisément ceux de l'Occident, ne signifie pas que le «temps occidental» (Laïdi, 1992) avance comme un rouleau compresseur, rencontrant sur son chemin un «homo-sociologicus éponge " (Padioleau, 1986), qui absorberait passivement tout ce qui traverse son champ mental : l'ouverture d'une région sur l'extérieur participe de la dynamique propre à cet espace. L'appropriation, l'une des modalités de cette interaction, se présente en deux temps : l'extraction d'un matériau de son environnement propre, spatial ou temporel, puis l'adjonction d'un élément d'une origine géoculturelle et historique distincte, ou l'attribution d'un nouveau sens, d'une autre structure. De cette opération, naît une production différente soit du point de vue de l'observable, soit de celui de la signification. Ce mécanisme règle un large pan des pratiques. Ainsi, à l'échelle du 
dialecte marocain, le terme utilisé peut subir une altération de sens, une transformation de structure, ou les deux à la fois. Pour répondre à de nouveaux besoins, contourner des tabous, ce vieux procédé fonctionne aujourd'hui informellement et de façon privilégiée avec les langues latines et anglo-saxonnes. À titre d'exemple, le terme "tbezniss", résultat de la dialectalisation sous une syntaxe active du mot anglo-saxon "business", renvoie à l'économie souterraine populaire et plus généralement à toute activité individuelle s'appuyant sur des ressources parallèles. Sur le plan musical, même en Égypte, berceau de la musique orientale contemporaine, une génération de chants «jeunes » emprunte sonorités et tempo au répertoire occidental. Au Maghreb, le processus est plus poussé : le rai, revivifié au cours des années quatre-vingt dans l'est marocain et l'ouest algérien, à partir du mixage d'un fond populaire ancien, de rythmes et d'instruments occidentaux, se fait l'écho des aspirations « jeunes » (Drouhet, 1990).

11 En circulant, la pratique s'enrichit. Ainsi, au Caire, le guide aura tendance à porter l'écharpe locale, par exemple, de la même façon que le visiteur: celle-ci n'est plus un élément faisant partie d'un ensemble, mais un ornement décontextualisé, accompagnant un jeans, des lunettes noires, etc. Un certain mimétisme se manifeste chez les jeunes Égyptiens, étudiants ou lycéens qui, durant leur temps de loisirs, se métamorphosent volontiers en touristes. En visite dans les hauts lieux de leur histoire, il leur arrive d'emprunter ne serait-ce que partiellement le style des Occidentaux de passage. Durant ces escapades, souvent en bandes, le port du hijâb par exemple - qui s'accommode aisément du jeans et des lunettes noires - peut se voir adjoindre casquette ou chapeau de paille. Si certains voyageurs aiment à prendre en photo l'habitant du pays - de préférence typé et en couleur locale -, le ou la jeune Cairote en promenade à la Citadelle ou ailleurs pourrait demander à l'étranger (ou à l'étrangère) de se faire prendre en photo avec lui ou elle.

12 C'est par la redéfinition que l'appropriation se traduit à l'échelle des représentations. Ainsi, pour la plupart des jeunes Marocains interviewés, le vêtement dit « occidental » est « leur », il est «normal », par opposition au hijâb, ou à l'habit «traditionnel ». De la même façon, les surprises-parties sont présentées non pas comme "occidentales » par contraste avec les fêtes locales, familiales, religieuses ou nationales, mais comme une activité «jeune ». À ce stade, la différenciation d'ordre géographique ne constitue pas un enjeu : d'autres clivages structurent l'univers du pensable.

Du point de vue de la réception des messages et des images, l'appropriation se manifeste par la réinterprétation, l'attribution d'une signification nouvelle. Face à un feuilleton américain ou même égyptien, les spectateurs «reconstruisent» individuellement ou collectivement le sens de ce qui leur parvient (Fiske, 1991 ; Liebes et Katz, 1990 ; Abu-Lughod, 1993, p. 25-30 ; El-Khawaga, 1997, p. 169-201). À travers les quelques cas évoqués, ce mécanisme se dévoile sous plusieurs aspects et révèle une mise entre parenthèses de l'altérité. Il arrive toutefois que cette logique continue à commander des dynamiques basées cette fois-ci sur une vision du dehors en tant qu'espace différencié.

\section{Attraction}

14 Pour les jeunes Égyptiens et Marocains, le degré d'attraction qu'exerce l'Occident est variable. Il arrive qu'il soit l'objet d'une fascination totale. Le cas limite consiste à 
magnifier cet univers non seulement par la valorisation de traits supposés être absents au sein du groupe d'appartenance, mais également de ceux stéréotypiquement considérés comme spécifiques à «l'ici». Pour une autre catégorie d'interviewés, le rapport à l'Occident, désigné en tant que tel, reste sélectif. La principale différence avec le premier groupe ne réside pas tant dans le contenu des qualités prêtées à l'Autre que dans la désignation simultanée de ce que l'on n'apprécie pas chez lui. Si l'Occident est construit en tant que bloc à partir de l'affirmation d'une prise de position de type idéologique, qu'il s'agisse d'ériger cet ensemble géographique en modèle, ou au contraire de le rejeter partiellement voire globalement, la vision de ('Ailleurs se fragmente dès lors que structuration d'ordre spatial et classification selon une échelle de valeurs cessent de coïncider. Ainsi, pour un certain nombre d'interviewés, ce n'est pas l'espace qui est objet d'appréciation, mais un élément qu'on y suppose présent. Et un tel jugement se fait sur un mode quasiment neutre. Il ne s'agit ni de disqualifier le groupe d'appartenance, ni de survaloriser l'Autre. À partir de «ce que l'on dit » et d'émissions documentaires télévisées, des personnes diront apprécier la France pour ses monuments, l'Allemagne pour son inventivité, l'Amérique et le Bahreïn pour leur opulence, la Suisse et les Émirats pour leur propreté, l'Italie, la Grèce, le Liban, la Tunisie pour leur beauté ou leur culture méditerranéenne...

Toutefois, y compris pour les chantres de l'Occident, celui-ci n'est pas un territoire indifférencié. En Égypte, ceux qui appréhendent cet espace en tant que bloc ont tendance soit à interchanger les vocables Amérique et Occident, soit à considérer les États-Unis comme le phare ou le moteur de ce grand ensemble. Par ailleurs, une hiérarchisation est régulièrement opérée en fonction des repères individuels de chacun. Pour Midhat, 30 ans, qui vit et travaille en Europe, l'ambiance et la gaieté animeraient davantage la Grèce, que la France ou la Suisse. Le critère de classification repose parfois sur une grille de lecture nationaliste, en rapport avec la mémoire du pays. Ainsi, on entendra souvent au Caire que les Français sont meilleurs que les Anglosaxons qu'il s'agisse de leur teint, du fait qu'ils aient des valeurs, parce que leur pays est un pays de liberté, de civilisation, de génie culturel, ou parce qu'ils font preuve de maturité en étalant moins les affaires de coeur de leurs dirigeants. Même la colonisation française est supposée avoir été plus enrichissante pour les Maghrébins que la britannique ne l'a été pour les Égyptiens. Selon 'Imâd, un Cairote âgé de 29 ans, diplômé de physique, les Français voulaient transmettre leur civilisation, leur langue, alors que les Anglais ne cherchaient qu'à extirper tout ce qu'ils pouvaient aux populations qu'ils ont soumises. L'éloignement de la France, la nature du fait colonial en Égypte semblent doter l'Hexagone d'une aura particulière. Au sein même des Anglosaxons, le Royaume-Uni est différencié, par rapport aux États-Unis, sur la base de son histoire, de son « authenticité ». Inversement, au Maroc, la France et les autres foyers d'accueil traditionnels (Benelux, Allemagne) connaissent une désaffection, par contraste avec l'Espagne, l'Italie, la Scandinavie, l'Amérique, le Canada, voire l'Australie. Le premier ensemble est accusé d'abriter le racisme à l'égard des Arabes et le second d'être plus hospitalier. La différence dans le degré et la nature de l'exposition à l'Occident contribue donc à éclairer ces dissemblances.

Parmi les jeunes d'un même pays, la proximité à l'égard de cet univers varie, entre autres, en fonction des canaux d'approche. Le jeune Casablancais qui fréquente le lycée français, qui appartient à la bourgeoisie francophone, qui a souvent l'occasion de se rendre à Paris, tout comme l'étudiant cairote qui fait ses études à l'université 
américaine, qui a visité New York ou qui y a vécu, n'ont pas le même réservoir d'images que les guides touristiques de Marrakech ou du Caire. Les entretiens effectués montrent cependant que si cette dimension agit sur la manière de nourrir l'argumentaire, elle n'en structure pas pour autant le contenu de la thèse défendue. Il semblerait que ce soit plutôt la trajectoire nationale et la dynamique sociale interne qui pèsent le plus dans la représentation de l'Occident qui fascine et des traits valorisants qui lui sont attribués.

$\mathrm{Au}$ Maroc comme en Égypte, l'Occident attrayant est en premier lieu une promesse d'exotisme. Des jeunes Marocains évoqueront vaguement la neige, la réputation de beauté de telle région, ses monuments, son architecture, les distractions qu'ils pensent trouver en Espagne ou à Las Vegas. Tel Cairote évoquera devant l'assemblée du petit café de Sûq Tawfîqiyya la fête du blé à Paris, la métamorphose de l'avenue des ChampsÉlysées et de la place de l'Étoile en champs de blé, décrites par son frère dans une lettre. À ce stade, l'Ailleurs ne fonctionne plus comme un détour, mais comme une source inépuisable de loisirs et de divertissements.

L'autre grande image en vogue est celle du développement, de la réussite technologique et économique. Le prolongement naturel de ce stéréotype cultive la représentation de l'Europe et de l'Amérique du Nord comme terres d'opulence, comme paradis du consommateur. Dans les kiosques des grandes villes marocaines, toutes sortes de revues françaises sont étalées. Celles-ci comportent des pages entières sur la vente par correspondance, à un prix modique, des gadgets dont les qualités pratiques et fonctionnelles sont vantées.

19 En fait, les notions qui s'accumulent sur l'Occident servent souvent de support à la production d'un discours sur le groupe. En dépit des apparences, ce n'est pas l'altérité qui en constitue le pivot, mais bien soi - le dehors étant un espace où se puisent des images dont l'instrumentalisation se soumet à une perpétuelle renégociation du sens en interdépendance avec l'«ici " (Somers, 1992, p. 591-630). C'est à ce niveau que se creuse le plus la différence de ton entre jeunes d'Égypte et du Maroc. Lorsque des thèmes sont partagés, c'est le degré de diffusion du stéréotype qui varie. Les qualités de l'individu et du citoyen dans sa relation à l'environnement et au groupe sont fréquemment louées. Pour 'Abdallah, un lycéen de Salé âgé de 19 ans, le civisme existe en Occident, mais pas au Maroc. Pour conforter son opinion, le second s'inspire de l'exemple d'un touriste qu'il a rencontré sur la plage. Alors que ses cousins et lui-même jetaient les pelures d'orange sur le sable, le Français les ramassait pour les mettre dans un sac en plastique. D'autres relèveront la ponctualité, la fiabilité, la rationalité de l'Occidental.

Plus que tout, ce qui serait inexistant « ici » et allant de soi « là-bas », c'est le respect de l'individualité du jeune et de la personne d'une manière générale. La dénonciation de la pression du groupe dans l'espace d'appartenance, qui s'accompagne d'une valorisation du contraire en Occident, traverse les catégories socio-économiques dans le cas des jeunes Marocains interviewés et semble plus circonscrite dans celui des jeunes Cairotes. Dans notre échantillon égyptien, ce point de vue est défendu par 'Azza, âgée de 22 ans, étudiante à l'université américaine du Caire, qui appartient à la grande bourgeoisie cairote et qui a vécu deux ans aux États-Unis, par Sânia, 32 ans qui réside dans un quartier informel, et qui connaît des difficultés conjugales, et enfin, plus largement, par ceux qui comme Midhat cherchent à se construire une légitimité en rapport avec l'Ailleurs. Cela va de la manière d'aimer, à la liberté sexuelle, en passant par les relations avec les parents. Munîr, un moniteur de Fès, âgé de 25 ans, se plaint de devoir 
fumer en cachette, de ne pouvoir ni écouter la musique qu'il apprécie, ni s'exprimer dans la maison parentale, alors qu'en Europe, " on est libre à 18 ans ». Au Caire, 'Azza ne comprend pas quant à elle que son père lui impose de rentrer le soir à des heures "convenables", à cause de ce que pourrait en penser le bawwâb (le gardien de l'immeuble) : «J'ai fait des études, j'ai vécu seule pendant deux ans en Amérique, et je devrais aligner mon mode de vie sur le système de valeurs du bawwâb.»

Dans les propos recueillis auprès de jeunes Marocains, et d'où ressortent un grand malaise, un sentiment d'insécurité et d'atomisation, une représentation de la vie sociale comme dérégulée, l'idée d'altérité est omniprésente ; elle structure l'ensemble des entretiens. La glorification de l'Occident constitue alors fréquemment le paravent à une contestation de tout ce qui compose le lieu d'appartenance. En fait, l'image de l'Autre se construit en étroite corrélation avec la perception de soi ; bien au-delà, l'Ailleurs reconstruit se présente comme un écran sur lequel se projette tout un ensemble d'attentes et de frustrations, et émerge ainsi comme un mode privilégié d'expression métaphorique. Ainsi, dans les interviews réalisées au Maroc, beaucoup plus qu'au Caire, l'Autre ne peut être qu'humain, consciencieux, droit, ouvert, modeste, tolérant, non médisant, direct, etc. L'entêtement, l'ostentation, la malignité, le tberguigu (le fait de s'attacher aux faits et gestes de l'autre, de médire) étant le propre du Marocain. Chaque trait formulé est suivi par son opposé attribué au "nous ». S'il y a encore une quinzaine d'années, le thème de l'absence de solidarité dans les sociétés occidentales était abondamment développé par opposition à l'hospitalité et à l'esprit communautaire prévalant au Maroc, depuis, la tendance s'est bien inversée : ce sont l'humanité et le désintéressement de l'«Occidental» qui sont opposés au "matérialisme individualiste» du Marocain. Su'âd, 25 ans, jeune fille voilée de Casablanca, va jusqu'à affirmer que le « vrai islam » n'existe pas dans son pays, mais en Occident. Cette thématique, qui traverse les écrits des réformistes musulmans dès le $\mathrm{XIX}^{\mathrm{e}}$ siècle, tendrait à se diffuser, voire à se banaliser. Lors des entretiens effectués en Égypte, là où l'on vante la prégnance des liens de proximité - notamment dans les quartiers "populaires» par opposition au centre-ville, aux lieux résidentiels des couches aisées du Caire, et par contraste à ce qui se produirait Ailleurs -, ce type de discours n'a été tenu qu'une seule fois. Sânia, dont le travail au Caire l'amène à fréquenter des Français, raconte que son lieu de voisinage idéal est un petit village français qu'elle a eu l'occasion de visiter! Du temps de sa mère, les voisins partageaient leurs plats, leurs joies et leurs peines. Aujourd'hui, ils hésiteraient à offrir un verre d'eau à un enfant qui a soif ; ils auraient tendance à jeter des détritus sur le passage du voisin.

Au Maroc, qu'il s'agisse d'idéaliser les partenaires sexuels potentiels ${ }^{6}$ ou les hommes politiques occidentaux, le détour par l'Autre met en relief des enjeux aussi bien que des valeurs en mutation (lesquelles ne sont pas pour autant des valeurs "occidentales »), mais où l'Occident intervient de biais dans la négociation de leur élaboration. La fascination exercée par la scène politique étrangère au Maroc est telle qu'elle mérite qu'on s'y attarde. En fait, l'ensemble des représentations que les jeunes se font sur d'autres lieux idéalisés du politique se structurent par les attentes et les espérances insatisfaites chez eux, et demeurent concrètement juxtaposées aux préoccupations et aux frustrations quotidiennes. C'est ailleurs, en Occident, que sont acquises les réalisations de l'État-providence, notamment la sécurité sociale et les indemnités de chômage. Aux grands écarts de fortune, au pouvoir de l'argent, au clientélisme, à la corruption régnant dans les rouages de la vie sociale, administrative et économique du 
Maroc, sont opposés la justice sociale, le « respect de l'homme » (sans considération pour sa condition sociale) régnant "ailleurs ", chez le voisin espagnol côtoyé dans les enclaves par les habitants du Nord marocain, en France, d'après les innombrables informations que véhiculent médias, émigrés et autres relations à l'étranger. C'est pour souligner l'étroitesse des marges de la "liberté » au Maroc, que l'on se réfère aux " étrangers ». Encore une fois, la conjugaison de la proximité et de l'éloignement prête à l'Ailleurs la possibilité d'agir comme un mode de désignation de ce qui est perçu comme dysfonctionnant dans l'« ici ». Cet univers rêvé se présente logiquement comme un espace de réinvestissement de toutes les aspirations.

Sur le plan politique, les jeunes Marocains manifestent un éloignement certain, affichant absence d'intérêt, crainte, désaffection à l'égard de la classe politique et érigeant cette activité en domaine réservé. Toutefois, la fuite de la scène politique marocaine "visible » se manifeste, entre autres, par un transfert dans une sphère du politique localisée ailleurs. C'est manifeste au niveau des représentations, mais également du point de vue de l'intérêt effectif porté à l'exercice du politique chez l'Autre. Cette fois-ci, l'Ailleurs englobe aussi bien des pays occidentaux déterminés (et avant tout les mieux connus, tels que la France et l'Espagne) que des pays arabes. Les jeunes Marocains sont davantage familiarisés avec les noms des hommes politiques français qu'avec ceux de la scène politique locale. Les élections présidentielles françaises suscitent un intérêt passionné (Ossman, 1991). Certains manifestent pour des mouvements politiques étrangers, une sympathie et un intérêt qui contrastent avec l'indifférence marquée à l'égard de leurs homologues au Maroc. Les socialistes en Espagne ou en France, les islamistes algériens, constituent des exemples contradictoires de "bons » modèles situés à l'étranger. Au sommet de ce processus, l'allégeance à des leaders charismatiques (Scott, 1990, p. 223) étrangers, tels Khomeyni de son vivant, Kadhafi, ou Saddam Hussein, cristallise une contestation aussi bien vis-àvis du système marocain, qu'à l'égard de l'Autre occidental. À ce niveau, l'Ailleurs est compartimenté en deux espaces, l'un «bon ", l'autre «mauvais ». Toutefois, s'ils sont nombreux au Maroc à avoir tendance à considérer que l'Occident bénéficie d'un système politique enviable, ceux-là n'en rejettent pas moins unanimement - comme on le verra - la relation jugée injuste qu'entretient ce bloc avec le monde d'appartenance. Par contraste, en Egypte, certains - comme Midhat ou comme un journaliste qui a séjourné en Israël -se dégagent tellement d'une identification au monde arabe et musulman, pour en général promouvoir l'égyptianité, qu'ils en viennent même à se désolidariser des Palestiniens ("la mère du monde », entendre: l'Égypte, aurait défendu la cause de ces derniers plus qu'ils ne l'auraient fait eux-mêmes). Il arrive que le propos soit vigoureux. Midhat considère ainsi Saddam Hussein comme un pitre ayant osé s'en prendre à plus puissant que lui pendant la guerre du Golfe. Dans cette configuration, on trouve logique que l'Amérique défende ses intérêts et on l'admire aussi parce qu'elle dirige le monde. Au cours de la dernière partie, nous tenterons de proposer une lecture de ces différences d'attitude. Quels que soient ses mobiles et son étendue, la fascination à l'égard de l'Occident se traduit parfois chez les jeunes Cairotes et très souvent dans le cas des jeunes Marocains par un rêve lancinant de départ. 


\section{Rêves de départ} projet dans le temps et dans l'espace, la nature individuelle ou collective du dessein
pourraient être en relation avec les modalités d'insertion aussi bien dans le tissu urbain que dans l'univers de la globalisation. Plusieurs personnes ne sont-elles pas susceptibles de partager un cercle, sans pour autant y jouer le même rôle, sans s'y impliquer, ni exploiter les occasions qu'il offre de la même manière? La réappropriation des quatre modes d'existence urbains dégagés par Ulf Hannerz (1983, p. 316-323) (enclavement, intégration, ségrégation, isolement) permet une relecture des différents types d'investissement dans le projet migratoire. Il s'agit en premier lieu de réajuster l'échelle, en appréhendant aussi bien la ville - comme espace comprenant des territoires de l'Ailleurs diffus ou localisés - que l'Ailleurs en tant qu'entités extraterritoriales. En liaison avec cet élargissement, il convient de redéfinir trois postures (enclavement, intégration, ségrégation) en leur adjoignant une quatrième : la non-intégration. Soulignons que ces positions constituent des idéal-types et qu'elles ne sont pas exclusives les unes des autres.

27 Selon l'auteur d'Explorer la ville, l'enclavement se caractérise par le regroupement de toutes les relations sociales significatives dans un seul ensemble, de telle façon que les rapports sociaux appartenant à des domaines dissemblables s'agencent de manière surdéterminée. La personne investit ainsi tout son temps et tous ses intérêts dans le même secteur. Il existe, dans ce cas, une très faible disjonction entre le rôle et le statut. Et l'individu «ne semble pas vraiment exploiter les occasions que lui offre la ville » (Hannerz, 1983, p. 317), ni celles, peut-on ajouter, que met à sa disposition l'Ailleurs supposé diffus dans son univers. Au cours de ses trajets et de sa trajectoire, du fait même de sa posture d'«enclavé », une partie de la ville, voire du café qu'il fréquente, est plongée dans la pénombre.

28 Dans une situation d'enclavement et face à la migration, trois types d'attitudes sont possibles. Pour Fathi, un jeune communiste âgé de 22 ans, qui travaille, vit et milite 
dans le Hayy al-sâbi' à Madînat Nasr ${ }^{7}$, il n'est pas question de quitter le quartier et encore moins l'Égypte. La seconde position consiste à concevoir le départ comme un projet délimité dans le temps - deux à quatre ans -, inscrit dans une approche utilitariste: études ou motivations strictement économiques. En ce qui concerne les interviewés marocains, ces dernières sont en net recul. Elles demeurent importantes dans le cas des Cairotes. Trouver un emploi mieux rémunéré, de meilleures conditions de travail, une chance d'intégration socio-économique plus rapide ou du moins plus sûre est le principal objectif de Sayyid, 30 ans, père de cinq enfants, qui réside et travaille comme boucher à Al-Wahda al-'arabiyya, à Shubra al-Khayma. Ce périmètre très dense du Caire est quadrillé par les familles notabilières qui ont bénéficié de la parcellisation et de la vente de leurs terres agraires. Plus généralement, pour les jeunes « enclavés ", il s'agit d'émigrer afin d'amasser un capital, retourner chez soi en bonne position pour créer une entreprise, se marier, acquérir un logement, voire une voiture. Le lieu de départ compte peu, pourvu que ce dernier fasse miroiter l'image de l'opulence. Selon Tawfîq, 26 ans, serveur au Pizza Hut, n'importe quel pays du Golfe, d'Europe, voire du Maghreb offre davantage de chances de trouver un travail rémunérateur que l'Égypte. Il arrive cependant que l'« enclavé » appréhende son projet sous forme d'installation. Le dessein est alors familial. Gamâl du Caire, 23 ans, travaille le bronze dans la même fabrique depuis ses douze ans. Rencontré dans le café du sûq Tawfiqiyya, il explique qu'il ne connaît aucune langue étrangère, qu'il ne fréquente pas les touristes, qu'il ne va jamais vers ceux-ci, qu'il les évite même. Soulignant sa différence avec d'autres jeunes, qui guettent en permanence les visiteurs en provenance de l'Ailleurs, il affirme que "lui » ne va pas vers " eux », que ce sont « eux " qui sont venus le chercher. Remarqué pour ses compétences manuelles par des experts allemands de passage dans la manufacture qui l'emploie, il s'est vu offrir un contrat et un billet d'avion, qui lui ont facilité l'obtention du visa. Pour Gamâl, il n'est pas question d'épouser une Occidentale; il en a une piètre opinion. C'est avec l'institutrice égyptienne qu'il fréquente qu'il se mariera. Il envisage également de faire venir en Allemagne sa mère, ses frères et sœurs.

Dans la perspective adoptée par Hannerz, l'intégration suppose quant à elle des relations significatives, un investissement du temps et des intérêts dans plusieurs domaines et secteurs, sans concentration notable sur l'un d'eux, et ce, avec une volonté - ou, en ce qui nous concerne, une capacité - d'organiser des rapports sociaux de nature différente dans un même tout (Hannerz, 1983, p. 320). Que ce mode d'insertion opère à un niveau strictement local, à celui de l'Ailleurs chez soi, ou qu'il englobe l'Ailleurs extraterritorial, il se définit notamment par son potentiel à réaliser une exploitation optimale des occasions et des ressources disponibles. Nombreuses sont les passerelles, intense est la mobilité. Quelle que soit sa position, celui qui est en situation d'intégration idéal-typique ne ressent pas le besoin de partir. Celui qui participe pleinement au « village global » ne se fait pas arrêter par les frontières: Paris, Londres, New York lui sont aussi accessibles que Marrakech, Alexandrie ou Assouan. Il arrive même qu'il dispose ici ou là d'une résidence secondaire. Ralat, 28 ans, fils d'un grand entrepreneur égyptien, ingénieur, formé à l'université américaine du Caire partage son temps entre le cabinet paternel qu'il dirige et les pubs des grands hôtels cairotes. À sa table sont rassemblés des amis d'enfance, des membres de la famille, mais aussi des connaissances étrangères. Affaires et loisirs les réunissent. Deux mois par an, Ra'fat séjourne dans la villa loridonienne familiale. Durant l'année, ses activités professionnelles le conduisent ponctuellement vers d'autres destinations. 
30 La situation de ségrégation apparaitt lorsque « deux ou plus de deux segments [d'un] réseau [...] demeurent bien distincts» (Hannerz, 1983, p. 319) sans superposition aucune. Ce mode d'existence intentionnel est - dans le cas limite - en rapport avec un " noir secret ». Il dépend de la capacité à préserver les distances entres les individus et les activités. À ce stade, la disjonction entre le rôle et le soi est totale. Dans notre cadre d'analyse, cette posture n'est pas ressentie comme un choix stratégique, mais comme une contrainte imposée par une composante du réseau : le cercle conjugal, les parents ou la famille, le voisinage, etc., toute autorité instituée comme légitime et dotée du pouvoir d'imposer des frontières dans le temps, entre les espaces, les trajets, les conduites, les fréquentations, entre le public et le privé, entre le visible et l'invisible, voire à forclore un pan de l'existence du sujet. Munîr de Fès comme 'Azza du Caire évoqués dans la partie précédente - se situent dans cette catégorie. Il existe aussi un niveau de "ségrégation " plus global. Ainsi, dans les ruelles de Bab Marrakech, dans la vieille médina de Casablanca, un jeune homme du quartier ne se permettra pas de poser le regard sur une jeune fille du quartier, aussi maquillée soit-elle, même si elle porte une minijupe et un décolleté. Inversement, lorsque celui-ci se déplace vers le centreville ou vers la corniche, lieux construits comme espaces de l'anonymat dérégulé, il se sent autorisé à draguer toute personne de l'autre sexe, abstraction faite du degré de « respectabilité » de la présentation publique de cette dernière. Toutes ces dissociations sont vécues dans le malaise, la tension et l'insécurité. Le plein épanouissement du soi passe alors par la négation de l'un des segments.

31 Le départ, vers une destination bien précise - en Occident - et pour une durée indéterminée, est, cette fois-ci, perçu comme une alternative qui s'accompagne d'un projet de vie: un emploi certes, éventuellement des études dans de meilleures conditions, mais aussi et surtout un espoir de réalisation totale de son individualité. Pour Saniyya, âgée de 32 ans, originaire du Caire, vivre en France, c'est connaître une société plus « courtoise ", plus « respectueuse de la personne », « moins malade », c'est s'affirmer loin des pesanteurs d'une société avide de contrôler les siens, tout en ayant cessé de leur offrir entraide et solidarité. Certes, Sânia dispose d'un réseau bien fourni dans son pays, d'un travail et d'un sens de la débrouille qui lui rapportent des revenus relativement corrects; mais elle décrit sa vie de couple comme un «enfer » et trouve peu de soutien auprès de ses parents auxquels elle tient rancœur depuis l'enfance. Son idéal est de reconstruire sa vie ailleurs. Mustafa, un dealer tangérois, âgé de 19 ans, voudrait également tout recommencer à zéro : études, sport, travail, mariage, etc. Il considère quant à lui qu'il aurait dû naître à 17 kilomètres, c'est-à-dire de l'autre côté de la Méditerranée; mais comme ce n'est pas le cas, il est disposé à corriger son mauvais sort en changeant nationalité et religion... Il n'est pas question, pour lui, d'épouser une compatriote. Celle-ci serait dotée d'une «multitude de facettes » et ne lui inspirerait aucune confiance. Partir équivaut alors à une rupture avec le groupe d'appartenance. Le riche répertoire marocain d'anecdotes ayant pour thème le départ fournit une illustration de cet état d'esprit : « Un tremblement de terre nocturne coupe le Maroc de l'Afrique et le pousse vers le continent américain. Un homme se réveille et prend conscience de ce qui se produit. Il se lève en silence, met un pied en Amérique et, avec l'autre pied, repousse le Maroc vers l'Afrique. »

La non-intégration est une catégorie intermédiaire entre les trois précédentes. Le nonintégré dispose d'une pluralité de réseaux dans différents secteurs. À l'inverse* de celui qui est dans une situation de ségrégation, ses relations sociales significatives se 
superposent ne serait-ce que partiellement. Il s'oppose à celui qui est dans une position d'intégration, par la difficulté qu'il éprouve à organiser avantageusement ses rapports sociaux dans un même ensemble, et notamment par la faiblesse de ses ressources et de ses opportunités. Sa posture se définit avant tout par la dissociation entre ses investissements, d'une part, et l'accessibilité aux objets de ses intérêts, d'autre part. Il existe plusieurs niveaux cumulables de non-intégration. Deux d'entre eux ont été observés. Le premier relève de l'échelle du groupe d'appartenance et comprend cercles familial, social et économique. L'individu se perçoit comme chassé de la trajectoire réussie d'intégration. Mustafa de Shubra al-Khayma, 30 ans, doté d'un diplôme de comptabilité, sans emploi stable, célibataire, cherche à partir n'importe où, pourvu qu'il trouve une meilleure opportunité de travail que celles que lui offre son pays. Il supporte d'autant moins sa situation que, tout en étant l'aîné d'une famille de dix enfants, il est considéré par son père comme un «bon à rien » et sa sœur cadette, une figure d'intégration locale réussie, le supplante au sein de la famille et du quartier, où elle joue un rôle de médiation très sollicité. Pour lui, émigrer c'est aussi tenter d'échapper à la marginalité et au regard des siens. La seconde position de nonintégration se distingue par un investissement dans les segments de l'Ailleurs chez soi et une accessibilité de cet univers (mesurable par la maîtrise des langues - plus précisément l'anglais -, le nombre des conquêtes occidentales pour les hommes, le réseau des relations étrangères), accompagnés d'une exclusion de l'Ailleurs extraterritorial.

Face aux innombrables difficultés qui se dressent sur le chemin du départ, trois types d'investissements se dessinent. Les «rêveurs » ne mettent pas en œuvre leur projet, ne serait-ce qu'en cherchant à obtenir un passeport et un visa : la conscience aiguë des obstacles au départ les maintient au stade de l'aspiration. Au coin d'une ruelle, dans un bistrot, seuls ou groupés, ils fantasment, échangent leurs informations, se projettent dans l'avenir: le rêve de départ, échappatoire en soi, participe alors à une renégociation de l'image sociale de celui qui le porte. Ils s'adonnent tout au plus à l'apprentissage et à la pratique des langues étrangères, cultivent leurs amitiés avec les Occidentaux de passage ou installés sur place. Au Caire, nombreux sont ceux qui rêvassent au mariage miraculeux avec une Occidentale. Le rêve peut prendre la forme d'une histoire d'amour avec un étranger ou une étrangère, rencontré(e) sur les lieux touristiques, dans un bazar, un bar, un hôtel ou les environs d'une pension. Échanges de lettres et de coups de fil prolongent l'idylle tout en offrant un support fantasmagorique au projet migratoire. Les rêveurs connaissent d'ailleurs beaucoup d'histoires qui ont débuté dans une boutique ou dans un café et qui ont fini par des noces, la conversion à l'islam de l'épouse et une installation dans un pays du Nord. Au Maroc, ce sont de plus en plus les jeunes filles qui nourrissent cet espoir.

Les «planificateurs » se distinguent par un projet clair, programmé dans le court ou le moyen terme. Ils ont déjà mis en œuvre leur plan ou disposent de différents éléments pour cela ${ }^{8}$. Ils ont une connaissance - très approfondie pour le cas des Marocains - des exigences administratives, ils ont réuni une somme d'informations et multiplié les relais parmi leurs relations déjà installées ailleurs, qu'ils s'agissent de concitoyens ou de touristes croisés lors de leur passage. Les jeunes Marocains essaient d'obtenir inscriptions universitaires, certificats d'hébergement et de prise en charge. Ils planifient jusqu'à leur insertion dans le pays de leur rêve, croyant disposer de réseaux pour obtenir un emploi saisonnier ou plus stable, dans la restauration par exemple, comptant le mariage avec une ressortissante du pays au rang des moyens d'intégration 
et de régularisation de la situation. Ils se repositionnent en outre par rapport au " marché » migratoire, en réévaluant la hiérarchie des destinations potentielles. Ainsi, la fermeture des frontières des foyers traditionnels de l'émigration, la France, le Benelux, ainsi que la conscience de l'amplification du phénomène de la xénophobie, font de ces espaces de simples relais obligés, du fait de la proximité culturelle et de la présence de réseaux. Pour certains, la solution consiste à se tourner vers les pays arabes. La plupart optent en fait pour de nouvelles Amériques : le Canada-francophone - l'Australie...

Contrairement à ce qui se produit au Maroc, en Égypte, l'idée que la crise a gagné l'Europe n'a pas encore fait son chemin, y compris chez ceux qui fréquentent quotidiennement des Occidentaux. Les interviewés cairotes sont beaucoup mieux informés sur l'épuisement des opportunités dans les lieux classiques de destination des émigrés égyptiens : les pays du Golfe, la Jordanie, l'Irak. Les conditions de retour des travailleurs pendant la guerre du Golfe continuent à marquer les esprits (Goshe, 1994). En prolongement du thème ancien du mauvais accueil réservé aux Égyptiens par les Arabes du Golfe, se diffuse l'idée que ceux-ci préfèrent désormais la main-d'œuvre philippine ou plus généralement sud-asiatique. On sait de plus en plus que nombreux sont ceux qui reviennent sans avoir rien accompli. Cela ne retient pas toutefois ceux qui veulent partir à tout prix. Mustafa de Shubra al-Khayma a sollicité toutes ses connaissances pour quitter son pays, où il avait une petite échoppe qui lui rapportait très peu. Il a fini par accompagner le mari de sa tante en Jordanie. Ses gains dans le pays d'arrivée n'étaient pas à la hauteur de ses attentes. En outre, il devait lui-même assurer son gite et son couvert, alors qu'au Caire il vivait chez ses parents. En dépit des pressions familiales, il n'a pas voulu revenir. Il a fallu que sa mère invente un subterfuge - maladie grave du père - pour qu'il prenne le chemin du retour. En Jordanie, Mustafa s'est aperçu que le pays traversait une crise économique. À partir de là, il s'était déjà mis en quête d'une nouvelle destination. Dès son arrivée en Egypte, il écrit à nouveau à toutes ses relations vivant en Occident ou originaires de cette partie du monde. Après avoir fait le deuil des pays arabes, il souhaite aller en Europe.

Pour d'autres, la configuration des lieux où ils projettent de partir est en étroite corrélation avec celui des endroits dont sont issues les relations étrangères qu'ils se sont faites le plus souvent au Caire. Gamâl a été remarqué pour son habilité artisanale par des Allemands. Pour 'Amr, le vendeur de souvenirs de Khân al-Khalîlî, qui a décroché un visa Schengen, la voie semble être facile. Diplômé de langue et civilisation espagnoles, il affirme connaître les dialectes et l'histoire d'Espagne comme si c'était les siens. En tant que guide, il a pu étendre et mettre en pratique son savoir. Il a par ailleurs souvent eu l'occasion de se rendre en Catalogne, où il s'est fait des amis. Là-bas, on le prend pour un fils du pays ou du moins pour un latino-américain. Grâce à son double réseau en Égypte et en Espagne, il pense pouvoir prospérer dans le commerce de parfum et d'objets exotiques, à Barcelone, où un ami est disposé à l'accueillir dans un premier temps. Dans le berceau du Flamenco, à celui qui se présente comme le Michael Jackson de la danse orientale, on aurait même suggéré d'ouvrir une école de danse. Pour obtenir le tampon tant souhaité et rejoindre son amie australienne établie en Grande-Bretagne, Ahmad, 25 ans, serveur dans un complexe touristique du Sinaï, compte sur la sympathie d'une Anglaise qui travaille dans une ambassade en Europe. Il n'en sollicite pas moins ses relations étrangères pour le conseiller d'une manière générale dans ses démarches, et parfois même sur ce qu'il doit vêtir avant de se présenter devant un consulat occidental. La réalisation du projet par les voies légales 
étant de plus en plus exceptionnelle pour des jeunes faiblement dotés socialement et économiquement, d'autres chemins sont empruntés, notamment au Maghreb.

Les « grilleurs » (Belbah, 1990) optent pour le viol des frontières fermées. Par centaines, des jeunes Marocains traversent clandestinement la Méditerranée sur des embarcations de fortune, nommées par la presse locale, les "barques de la mort », et ce, grâce à des réseaux qui organisent leur départ contre une somme d'argent. Certains se noient à l'arrivée, d'autres sont arrêtés par les autorités espagnoles et une partie parvient à s'infiltrer. Le fait que la majorité de ces jeunes soient conscients que l'Ailleurs n'est pas toujours un Eldorado ne freine pas leur désir de partir'.

Ces éléments confortent la position de ceux qui résistent à l'appel de l'Occident. En Égypte, ils construisent leurs arguments sur la base du rejet de l'lnfitâh, de la société de consommation, de la "conquête culturelle », ou sur celle du devenir des enfants nés d'un mariage mixte et élevés en terre étrangère. Au Maroc, ils se fondent sur la montée de la xénophobie, sur les expériences malheureuses des Maghrébins clandestins et clochardisés. Mais en réalité, ici et là, ces dires ont pour point de départ un mode d'insertion, des interactions au cours desquelles se construisent les positions, se consolident les légitimations, se négocient les valeurs et les frontières mouvantes. Dans le cas égyptien, la fréquentation - pendant quelques soirées - d'un café situé près d'une pension de touristes et d'un souk du centre-ville du Caire, m'a permis de dégager quelques observations. La plupart des figures esquissées étaient présentes. Mamdûh, 32 ans, pourvu d'une carrure impressionnante, héritier du nassérisme, et avant tout d'une branche familiale de Haute-Égypte, est propriétaire d'une boutique de sport. Il est également doté d'un diplôme en sciences religieuses de l'université d'Al-Azhar. Il maitrise l'anglais, mais évite de l'utiliser. Sans doute, préfère-t-il mettre en valeur son ancrage dans une région et dans une famille qui se distingue par sa taille, par son importance économique, par son ancienneté dans le lieu d'accueil et d'origine, et par son rôle dans la gestion des conflits internes. Mamdûh, personnage respecté et écouté du café, se moque ouvertement des rêveurs, des planificateurs, des "grilleurs" potentiels, que ceux-ci soient dans une position de ségrégation ou de non-intégration. Midhat, de retour de Suisse où il vit dans une situation précaire, essaie souvent de contrer Mamdûh, en chantant la gloire de l'Amérique et de l'Occident. Fawzî, qui semble se mouvoir dans le monde de la ségrégation, s'efforce quant à lui d'occuper le devant de la scène en se présentant au café avec un beau costume et un gros cigare. Un enfant qui vend des mouchoirs en papier le prend pour un pacha. Fawzî en profite pour raconter qu'il possède un commerce fructueux d'œufs et pour s'étendre sur son voyage à l'étranger, agrémenté de la compagnie d'une amie allemande. Après son départ, ses détracteurs l'accusent de se faire entretenir par un Allemand et d'arrondir ses fins de mois par des activités de prostitution masculine et de proxénétisme. Un soir, le jeu de cartes fait éclater une dispute tout en révélant des rancœurs et des clivages. Gamâl qui est sur le point d'émigrer en Allemagne s'est fâché avec Hasan, la jeune vedette du café et du souk, qui parle anglais, collectionne les conquêtes occidentales, tire des bénéfices de son business avec les touristes de la pension, épiés à partir du commerce de fruits paternel. Gamâl s'est également battu avec Ahmad, le serveur du Sinaï, intégré dans l'Ailleurs chez soi, selon le même modèle que Hasan, mais refoulé par les représentants officiels de l'Ailleurs extraterritorial. À partir de cette date, Gamâl et ses compagnons se liguent contre Ahmad, Hasan et leurs amis du souk. La parole de l'ouvrier-artisan se libère. Il ne rate plus une occasion pour souligner son appartenance au groupe des fallâhin (paysans) du Bas-Delta, (Hasan et les commerçants du souk sont originaires d'un 
village de Haute-Égypte), tout comme il tire orgueil de ce qui constitue sa position d'enclavé et de ce qui fait sa différence avec ses adversaires (son ignorance des langues étrangères, son évitement des touristes, son savoir-faire manuel qui lui a valu un visa et un contrat en Allemagne). L'ensemble de ces modes d'insertion sous-tendent largement les arguments développés lors des réquisitoires contre l'Occident.

\section{Répulsion}

Alors que l'idéalisation de "l'Occident» se fait parfois au détriment de l'espace d'appartenance, sa diabolisation repose en général sur l'expression de l'identification de l'individu à «sa » communauté, cette fois auréolée et définie en fonction de critères géographiques, linguistiques, historiques ou religieux : la marocanité ou l'égyptianité, l'africanité, mais surtout l'arabité et l'islam. Selon les mêmes procédés d'instrumentalisation observés dans le processus de valorisation de l'Ailleurs, le rejet de celui-ci s'exprime essentiellement en fonction d'appréciations de type éthique ou se construit sur une vision des rapports de pouvoir à l'échelle mondiale ${ }^{10}$.

Les thèmes de l'authenticité, de la sauvegarde des valeurs qualifiées tantôt de marocaines ou d'égyptiennes, tantôt d'arabo-islamiques, et renvoyant le plus souvent à la sphère des mœurs et de la sexualité sont largement présents ici et là. Au Maroc comme en Égypte, est dénoncé le matérialisme attribué à l'Occident. Dans le royaume, les jeunes interviewés, qui soulignent souvent que ce mal ronge leur société, ajoutent que l'enjeu consiste à se démarquer par rapport au modèle culturel « importé ", afin de renouer avec les fondements de l'idéal musulman. Dans la République, cet état d'esprit est, dans la plupart des entretiens, opposé à l'«âme égyptienne », présentée comme encore prégnante. En prolongement de ces arguments, la question de la désintégration de la famille occidentale est particulièrement présente dans les propos des jeunes cairotes et ce, comparativement à une société nilotique qui se distinguerait par une grande solidarité sociale, par l'importance et la stabilité de la famille. Certains ne manquent pas de mettre cela en rapport avec l'ancienneté de la civilisation et du développement social en Egypte: « $\mathrm{Si}$, pour nous, le développement économique et politique est difficile à réaliser, pour eux, le développement social est aussi ardu à concrétiser. "

41 Tandis qu'au Maroc, l'on dénonce l'aliénation «constatée » des Marocains et l'« impérialisme culturel » des Occidentaux, en Égypte, les nostalgiques du nassérisme manifestent la crainte de voir leur pays «souillé » par la conquête culturelle occidentale, réputée avoir été facilitée par le président Sadate et sa politique d'ouverture économique et politique. Les jeunes qui « rappent ", l'affluence dans les MacDonald's, seraient autant de signes de cet envahissement, qui sèmerait insidieusement les graines de la désintégration sociale, qui creuserait les écarts de fortune, qui détruirait l'esprit de groupe ${ }^{11}$.

42 Racisme, fermeture des frontières, absence d'hospitalité constituent un autre grand pôle. Si pour certains jeunes cairotes, la difficulté d'obtenir un visa est vécue à l'échelle individuelle (compte bancaire insuffisant, etc.) et ne débouche pas sur le développement d'une rancœur à l'égard de l'Occident (El-Khawaga, non-publié ; Amin, 1989, p. 103-121), pour d'autres, c'est la voie ouverte au dépit. Ahmad, le serveur du Sinaï, a été profondément humilié par l'accueil qu'on lui a réservé dans un consulat européen. Pour lui, la non-réciprocité entre l'Occident et l'Égypte relève d'un profond 
déséquilibre : «Ils viennent chez nous quand ils veulent, on les reçoit comme des rois. Et eux, ils nous claquent la porte au nez. » Ce type de discours ne se retrouve en Égypte que chez une partie des jeunes, notamment chez ceux qui sont dans une situation de non-intégration, à l'échelle de l'Ailleurs extraterritorial. Cela n'est pas sans relation avec le fait que la migration égyptienne vers l'Occident a pendant longtemps été le fait des couches moyennes ou des élites, alors que la main-d'œuvre bon marché constituait un réservoir pour les pays arabes pétroliers (El-Khawaga, non-publié). Au Maroc, en revanche, ce langage est largement répandu, et ce y compris au sein de la jeunesse dorée. Toute manifestation de xénophobie, les faits divers impliquant des immigrés, occupent la première page dans la presse d'opposition marocaine, et retentissent intensément dans les esprits. Le " phénomène Le Pen » excite la rumeur, le danger de ses succès pour les « Arabes » est particulièrement amplifié. C'est bien la proximité en elle-même et l'ancienneté de la migration maghrébine en Europe qui alimentent à la fois les sentiments de menace, d'inquiétude, de rejet par l'Autre, en liaison avec la représentation de l'Ailleurs comme source d'agressions envers le groupe d'appartenance.

Lorsqu'il s'agit d'appréhender l'Occident dans ses relations avec le monde arabe et musulman, de nombreux éléments de discours sont partagés par les interviewés au Maroc et au Caire. Il est toutefois frappant de noter la relative unité de ton des Marocains à ce sujet, par contraste avec la diversité des grilles de lecture des Cairotes. L'Occident, et à sa tête l'Amérique, "maître de l'univers ", sont considérés comme une calamité lorsque la personne se situe, à partir d'une identification au groupe d'appartenance - défini par l'égyptianité ou la marocanité, l'arabité, l'islam, ou même le tiers-mondisme -, dans un rapport de pouvoir défavorable aux siens. Du présent, jeunes Marocains et Cairotes remontent vers le passé. Aux croisades et à l'épisode colonial, ces derniers ajoutent l'agression napoléonienne, le pillage des trésors égyptiens, les guerres menées par les coalitions occidentales contre l'Égypte durant le $\mathrm{XX}^{\mathrm{e}}$ siècle. Les Égyptiens déplorent être réduits à des êtres sous-développés par les Occidentaux, « et pourtant, au temps où l'on construisait les pyramides, ils en étaient à l'âge de pierre ». Ici et là, s'est diffusé le sentiment selon lequel l'Occident a construit le monde arabo-musulman comme ennemi et cible, après l'effondrement du bloc de l'Est. Le premier épisode de cette nouvelle série est la guerre du Golfe. Concernant cet événement, les Marocains - à l'exception de leur souverain - ont adopté une position quasi-consensuelle.

Les moments de crise, qui agissent tels des révélateurs, montrent qu'au Maroc, plus que la proximité, la fascination et la volonté d'appropriation conjuguées à la fermeture de l'espace rêvé peuvent susciter haine et violence. Pendant la guerre du Golfe, notamment durant les mois de janvier et février 1991, c'est en termes de fureur que le rejet latent se manifeste. Si les consulats et les ambassades n'ont pas été directement attaqués comme dans d'autres pays arabes, des drapeaux américains ou français ont été brûlés, l'Occident - entre autres - a été conspué dans des manifestations quotidiennes, rassemblant cette fois toutes les couches de la société. Les slogans, les banderoles ont véhiculé des images négatives de l'Occident. Tous les répertoires - arabité, islam, tiersmondisme - ont contribué à nourrir cette dynamique de la diabolisation : colonialisme, impérialisme, sionisme se relient et se correspondent dans la lecture des relations entre l'Occident et les Arabes durant l'histoire contemporaine. Encore une fois, la remontée se prolonge jusqu'aux croisades, pour caractériser le rapport à l'espace qualifié haineusement d'« ennemi». Que lui reproche-t-on? Injustice, politique du 
«deux poids, deux mesures», usage d'un subterfuge juridique, «la légitimité internationale ", pour servir des intérêts « impérialistes ", défense d'Israël qui, depuis sa naissance, "viole les lois onusiennes ", «écrasement militaire d'un Arabe qui a osé se révolter contre l'ordre occidental, dont l'un des principes est le maintien des Arabes dans l'humiliation et l'exclusion ». C'est la non-redistribution de la richesse, la légitimité historique qui justifieraient l'acte de Saddam Hussein. Les enjeux véritables de la guerre seraient la libération du Golfe d'une présence étrangère profanatrice d'un espace sacré, les Lieux Saints, et encore davantage la libération de la Palestine, plaie ouverte du nationalisme arabe. En fait, c'est dans l'imbrication des trois dynamiques qu'il convient de rechercher une lecture de ces éléments de discours.

Un Occident que l'on pense s'être approprié, un Occident fascinant, un Occident exécré. Ces postures coexistent ou se succèdent, mais n'obéissent en aucun cas à une attitude systématique ou globalisante : ce ne sont pas les mêmes populations qui entretiennent sans discontinuité un rapport donné à un espace déterminé. D'une façon générale, une pluralité de répertoires sont investis et instrumentalisés dans un processus dynamique, traversé tantôt par une logique syncrétique - au moins deux références appartenant à des répertoires distincts sont ramenées à un même contenu et mobilisées au service d'un argument ou d'une représentation -, tantôt par une logique éclectique - un même espace ou répertoire faisant l'objet de représentations apparemment opposées. Dans le rapport des jeunes Marocains à l'Occident, c'est cette dernière approche qui intervient de façon privilégiée. Loin d'être aléatoire, son fonctionnement obéit à une matrice donnée qui embrasse à la fois le national et le transnational, et qui commande le compartimentage et la variabilité même des contours de l'Ailleurs.

En temps normal, les jeunes Marocains, qui sont dans une situation de non-intégration - que ce soit à l'échelon local, à celui de l'Ailleurs chez soi, ou à celui de l'Ailleurs extraterritorial -, cherchent à combler la distance qui se trouve entre leurs investissements et les objets de leurs intérêts, à s'insérer dans un système qui a du mal à les absorber, en usant de toutes sortes de tactiques individuelles. Afin de sortir de leur marginalité, ils multiplient précisément les tentatives de gagner un Ailleurs qui se ferme de plus en plus à eux. En temps de crise, que ce soit pendant les émeutes de décembre 1990 notamment à Fès et à Tanger ${ }^{12}$ ou pendant la guerre du Golfe, leur violence est d'abord un mode de participation, puis la formulation d'un rejet, non pas $\mathrm{du}$ système international et national en soi, mais celui d'un univers qui les repousse ${ }^{13}$. Les différents types de manifestation de mécontentement pendant la guerre du Golfe portent également la marque du sentiment d'exclusion, mais la ligne de rupture se déplace. Cette fois-ci, l'ensemble de la société se perçoit comme appartenant à un monde d'exclus; les termes étant de nature pluridimensionnelle, cumulative, puisés aussi bien dans le répertoire du nationalisme arabe, de l'islam que celui du tiersmondisme. Le clivage sépare dans ce schéma les «Arabes » et les «musulmans » des «Occidentaux, soutien d'Israël »; les pays du Tiers-Monde seraient maintenus dans la dépendance économique, politique et militaire, des "puissants» de ce monde. L'alliance du roi Hasan II avec «l'ennemi " favorise la transposition du schéma de pouvoir prévalant à l'intérieur du pays sur celui des rapports de forces construits à l'échelle transnationale. Dans cette équation, le leader - Saddam Hussein en l'occurrence - est le représentant des bafoués auxquels ces derniers s'identifient dans le défi qu'il lance à un adversaire plus puissant que lui, qui lui refuse l'équité dans le traitement, qui lui renie le droit à l'armement et au rééquilibrage des richesses, qui le trahit I Là, survient l'autre ligne de déplacement par rapport au schéma de la crise 
sociale. Le 14 décembre, ce sont les marginalisés qui expriment leur rancœur à l'égard de l'« ici » et de l'Occident qui les « rejettent; pendant la guerre du Golfe, les membres des classes moyennes et des couches supérieures - ceux qui se pensaient dans une situation d'intégration aussi bien dans leur pays que dans le monde - se joignent à eux, se sentant stigmatisés, abandonnés par un système qu'ils pensent s'être approprié, mais qui les bannit, notamment en s'alliant à leur « ennemi » et en pratiquant avec eux ce qu'ils perçoivent être une politique du «deux poids, deux mesures». Ce schéma rejaillit lors de chaque confrontation entre les "puissants" de ce monde et les « humiliés », arabes ou musulmans.

Le quasi-unanimisme dont ont fait preuve les Marocains durant la guerre du Golfe n'a pas son équivalent en Égypte (Roussillon, 1990). Si certains ont défendu la position des États-Unis, considérant que Saddam Hussein n'a obtenu que ce qu'il a cherché en défiant plus puissant que lui et en menaçant les intérêts vitaux de la première armée mondiale, d'autres ont plutôt relevé les aspects conflictuels de la dynamique régionale, mettant l'accent sur les rivalités et les rancoeurs des protagonistes arabes en présence. Y compris parmi ceux qui étaient défavorables à l'intervention américaine, les positions n'étaient pas unifiées. Le journaliste, qui au dénouement de la crise a décidé d'apprendre l'hébreu et de se rendre en Israël, était pourtant contre l'intervention de l'Égypte aux côtés des États-Unis et de l'Arabie Saoudite pendant la guerre du Golfe. Selon lui, « les intérêts » de l'Égypte commandent que le berceau des pharaons opère une "rupture épistémologique", se dégage de son environnement culturel arabe quitte à adopter l'anglais comme première langue d'enseignement -, et établisse des relations équilibrées avec toutes les forces régionales : Irak, Israël, Iran... Son souci premier en 1991 était de ne pas rompre l'équilibre du Moyen-Orient, en défaveur de l'Égypte, par l'apport d'un soutien à une force plutôt qu'à une autre. Tout en condamnant la destruction de la plus importante armée arabe par une puissance occidentale, des interviewés étaient contre l'incursion irakienne au Koweït. On ne peut pas s'en prendre à Israël parce qu'il occupe des terres qui ne lui appartiennent pas et en même temps approuver la violation du droit international par un président arabe, estime une militante pro-palestinienne de gauche de l'université de 'Ayn Shams, âgée de 23 ans. Pour un jeune commerçant de Sûq Tawfîqiyya, très friand de business avec les touristes occidentaux, Saddam Hussein s'est conduit comme un «idiot ». Il avait une bonne raison de s'attaquer directement à Israël qui a détruit sa base nucléaire en 1981. $\mathrm{Au}$ lieu de cela, il s'en est pris à un État pétrolier paisible et prospère, qui ne bénéficie pas d'une organisation en vue de la guerre. D'autres, enfin, ont soutenu Saddam Hussein, justement parce qu'il a défié plus puissant que lui, qu'il a menacé Tel-Aviv. Certains ajoutent que cela n'aurait été que justice que la gestion des richesses arabes revienne à ce leader plutôt qu'aux monarques de la région. Aux yeux de ceux qui ont condamné le rôle joué par les Américains, «défenseurs d'Israël» ou «suppôts du nouvel ordre mondial juif », l'affaire bosniaque a confirmé que l'interventionnisme de la puissance mondiale a pour corollaire l'attentisme et le laisser-faire lorsque les intérêts de celle-ci ne sont pas en jeu (Farag, 1996, p. 41 -50). De là à construire un monde polarisé confessionnellement, il n'y a qu'un pas, vite franchi par certains.

Comment interpréter le consensus marocain face à l'émiettement de la construction des significations chez les jeunes Cairotes ? Les modes d'insertion dans l'« ici » et dans l'Ailleurs offrent une première grille de lecture. Au Maroc, la proximité et le désir d'Occident semblent beaucoup plus intenses qu'en Égypte. Ce sont, cependant, les dissemblances au niveau des trajectoires nationales respectives qui peuvent contribuer 
à nous éclairer sur l'interprétation collective du conflit par les Marocains dans des termes historicisés, opposant un "nous" arabo-musulman, à un ennemi occidental. L'expérience $\mathrm{du}$ nationalisme arabe et des leaders charismatiques est fondamentalement différente, ici et là. L'arabisme a un fort retentissement dans l'imaginaire des jeunes Marocains, mais le Machrek reste lointain. Les interviewés cairotes ont vécu, de plusieurs manières, l'implication active de l'Égypte dans les affaires arabes. Nombreux parmi eux sont ceux qui n'en retiennent que les guerres, les pertes humaines et matérielles. Certains affirment que si la «mère du monde » s'était davantage préoccupée de ses intérêts, au lieu de s'investir dans un rôle régional, elle ne serait pas aujourd'hui comptée dans les rangs des pays sous-développés. Beaucoup d'interviewés, y compris de sensibilité nassérienne, défendent l'idée qu'il n'y a plus qu'une seule guerre à mener : celle de l'économie. Saddam Hussein est par ailleurs un leader du type de Gamâl Abdel Nasser. Un sentiment de lassitude face au charisme de ceux qui « osent défier " traverse bon nombre d'entretiens effectués au Caire. Pour un jeune marxiste, dont l'engagement s'est pourtant cristallisé pendant la guerre du Golfe, il vaut mieux pour le pays être gouverné par un fonctionnaire - Hosni Mubarak - qui se contente de gérer des dossiers, plutôt que d'être conduit par de vrais leaders politiques qui, dans l'histoire récente, n'ont mené l'Égypte que vers les catastrophes. Les jeunes Marocains quant à eux n'ont connu que Hasan II. Et, souvent, ils considèrent qu'un chef comme Nasser ou Saddam constitue une alternative au régime monarchique. Par ailleurs, le rapport aux pays arabes pétroliers contraste ici et là. Au Maroc, la péninsule Arabique symbolise souvent la richesse non-méritée et non-redistribuée. En outre, ses monarques sont associés au souverain alaouite. Celui-ci est aussi accusé d'avoir ouvert les portes du Maroc aux Saoudiens et aux Koweitiens, de les avoir autorisés à venir commettre les turpitudes sexuelles prohibées chez eux. En ce qui concerne l'Égypte, les pays pétroliers ont pendant longtemps été des destinations privilégiées pour les candidats à l'émigration. Les Égyptiens n'ont pas beaucoup de sympathie pour les Arabes de la péninsule; mais, l'écho des misères endurées par les travailleurs égyptiens en Irak, les pertes matérielles que ceux-ci y ont subi pendant la crise du Golfe ont largement diffusé un sentiment anti-irakien. Ces différences de configurations nationales se sont traduites par des réactions dissemblables lors du conflit du Golfe, qui a pourtant constitué un moment fondateur dans les rapports entre Occident et monde arabe.

Depuis cet événement se cristallise, ici et là, l'idée que le monde musulman est stigmatisé et que l'Occident multiplie les coups montés pour le détruire. En Égypte comme au Maroc, des interviewés affirment que si l'extrémisme religieux a été encouragé par les États-Unis par le biais de l'Arabie Saoudite et de l'Afghanistan, le terrorisme et la violence ont directement ou indirectement été suscités par les grandes puissances dans le but de nuire. Par ailleurs, d'après des jeunes du sûq Tawfiqiyya, la violence en Algérie serait le fait de l'interventionnisme français : la France a refusé à un pays le droit de choisir ses gouvernants. Pour d'autres, les Occidentaux amplifient tout événement, aussi minime soit-il, pourvu que celui-ci avilisse l'image de l'islam, qu'ils veulent associer au terrorisme dans l'esprit de tous. Parfois, plusieurs raisonnements se bousculent dans un même entretien. Un fonctionnaire de Shubra al-Khayma défend, dans un premier temps, l'opinion selon laquelle l'organisation internationale du Jihâd souhaite agir en vue de renverser les régimes corrompus pour instaurer l'islam. Puis, il affirme que les membres de ce mouvement sont étrangers au déferlement de la 
violence et que celle-ci est plutôt alimentée par l'Occident qui cherche, sciemment, à diviser les musulmans et à en finir avec l'islam qui lui fait peur.

La fascination exercée par un espace qui ferme ses frontières détermine en grande partie sa diabolisation. Au Maroc, voire en Égypte, le sentiment que l'Occident se verrouille tout en exhibant ses attraits - notamment par le biais du tourisme et de la parabole - suscite des tensions. Le sentiment de non-intégration, surdéterminé par la perception d'une stigmatisation, voire d'un rôle imposé - l'islam construit par «l'Occident » comme nouvel ennemi après l'effondrement du bloc de l'Est - conduit par moment la personne ou le groupe à se situer dans un rapport conflictuel historicisé remontant aux croisades, en passant par les épisodes coloniaux et trouvant leur aboutissement dans la guerre du Golfe et le massacre des Bosniaques. Ce schéma prédomine au Maroc face à une tendance, représentée essentiellement par le pouvoir et les grands entrepreneurs, qui affirme timidement la vocation du dragon chérifien, en voie d'intégration au marché européen. En Égypte, la construction hostile des relations entre l'Occident et l'islam rivalise avec une vision désabusée du rôle arabe de l'Égypte : «La mère du monde a trop donné, trop souffert. » Dans cet esprit, une partie des interviewés cairotes chante les louanges de l'Infitâh, considère que la paix avec Israël est une bonne chose, et que Saddam Hussein s'est ridiculisé.

Soulignons, encore une fois, qu'il existe une correspondance entre, d'une part, le mode d'insertion dans la ville et dans le monde de la globalisation, et d'autre part, l'intensité de l'attraction exercée par l'Ailleurs. L'individu en position de non-intégration ou de ségrégation est plus tenté par le départ, comme alternative ou nouveau projet de vie, que celui qui est dans une situation d'enclavement ou d'intégration. Plus on est ancré dans son groupe, moins on envisage une migration autre que ponctuelle. Plus l'Ailleurs est vivable chez soi, moins la tentation de partir est grande. Les privilèges socioéconomiques atténueraient les frontières, doteraient les individus d'un grand pouvoir de mobilité.

Comparativement au Maroc qui paraît beaucoup plus ouvert sur l'Occident, l'Égypte est souvent présentée comme une société où de fortes régulations sociales freinent aussi bien l'affirmation individuelle que l'appel de l'Ailleurs, suscité par des motivations autres qu'économiques. Or, les enquêtes effectuées nous ont permis de réaliser que les phénomènes observés au Maroc trouvent leur équivalent au Caire, notamment chez les jeunes évoluant au contact du tourisme et de son industrie. Dans la mesure où l'accès à l'Occident constitue une ressource potentielle - au moment même où les opportunités offertes par les pays du Golfe se tarissent -, celui-ci intervient, dans certaines circonstances, dans la structuration des relations au sein du groupe; il fonde aussi des rivalités qui s'accompagnent de négociations de valeurs, en relation avec les nouvelles fragmentations socio-économiques produites par une politique d'Infitâh ayant engendré de nouvelles couches sociales et sources de richesses. L'articulation, sous un mode comparatif, de trajectoires nationales et de modes d'insertion individuels, contribue à mesurer autrement l'impact produit par l'Ailleurs. 


\section{BIBLIOGRAPHIE}

ABU-LUGHOD Lila, 1993, « Islam and Public Culture: The Politics of Egyptian Television Serials », Middle East Report, January-February.

AMIN Galal A., 1989, « Migration, Inflation and Social Mobility: A Sociological Interpretation of Egypt's Current Economic and Political Crisis », in TRIPP Charles, OWEN Roger (Eds.), Egypt Under Mubarak, London and New York, Routledge.

BELBAH Mustapha, 1990, Les nouveaux immigrés, le cas nantais, Mémoire de DEA, Paris, Institut d'Études Politiques.

BENNANI-CHRAÏBI Mounia :

- 1994, Soumis et rebelles, les jeunes au Maroc, Paris, CNRS Éditions.

-1997, « Le Maroc à l'épreuve du temps mondial », dans LAÏDI Zaki, Le Temps mondial, Bruxelles, éd. Complexe.

DEPAULE Jean-Charles, 1990, « Des territoires en formation : jeunesse et urbanisation au Caire », Égypte/Monde arabe, $\mathrm{n}^{\circ} 1$.

DROUHET Véronique, 1990, Le Raï de l'Algérie à la France. La réinterprétation d'un phénomène musical par les jeunes issus de l'immigration, mémoire de DEA, Paris, Institut d'Études Politiques.

FARAG Iman, 1996, « Ces Musulmans de Tailleurs : la Bosnie vue d'Égypte », Maghreb-Machrek, $n^{\circ} 151$.

FARGUES Philippe, 1994, «Explosion démographique ou rupture sociale ? dans SALAMÉ Ghassan, Démocraties sans démocrates, Paris, Fayard.

FISKE John, 1991, Television Culture, Londres, Routledge.

LIEBESTamar, KATZElihu, 1990, The Export of Meaning, Cross-cultural Readings of Dallas, New York, Oxford, Oxford University Press.

GOSHE Amitav, 1994, Un Infidèle en Égypte, Paris, Seuil.

HANNERZ Ulf, 1983, Explorer la ville, Paris, Éditions de Minuit.

EL-KHAWAGA Dina :

- 1997, « Le détour par le Japon : un regard égyptien sur le temps mondial », dans LAÏDI Zaki, Le Temps mondial, Bruxelles, éd. Complexe.

- « Rester, partir, revenir ? Des Égyptiens au carrefour des temporalités », rapport pour le groupe « Temps mondial », Paris, CERI, non-publié.

LAÏDI Zaki (dir.), 1992, L'ordre mondial relâché, Paris, Presses de la FNSP.

LAROUI Abdellah, 1987, Islam et modernité, Paris, La Découverte.

OSSMAN Susan, 1991, Moving Pictures : Mass Images and Society in Morocco, Ph.D. Dissertation, Berkeley, University of California.

PADIOLEAU Jean G., 1986, L'ordre social, Paris, L'Harmattan.

ROUSSILLON Alain, 1990, «L'opposition égyptienne et la crise du Golfe : Saddam Hussein, ou comment s'en désolidariser », Maghreb/Machrek, $\mathrm{n}^{\circ} 130$. 
SCOTT James C, 1990, Domination and the arts of resistance. Hidden transcripts, New Haven, London, Yale University Press.

SOMERS Margaret R., 1992, « Narrativity, Narrative Identity, and Social Action: Rethinking English Working Class Formation », Social Science History, 16(4).

VATIKIOTIS P. J., (1969) 1991, The History of Modem Egypt : From Muhammad Ali to Mubarak, The Johns Hopkins University Press, 4th Ed.

\section{NOTES}

1. Les enquêtes de terrain conduites au Maroc et au Caire nous ont permis d'aborder ces dynamiques. Ici et là, nous avons adopté les méthodes de l'observation, ainsi que celles des entretiens non directifs et semi-directifs. Pour le cas marocain, l'étude a été menée dans un cadre plus large (voir Bennani-Chraïbi, 1994). Deux enquêtes exploratoires ont eu lieu en février et en avril 1989, auprès d'une vingtaine de jeunes, à Casablanca et à Rabat. Une trentaine d'interviews ont été réalisées, pendant l'été et l'automne 1989, à travers 12 villes marocaines. Un échantillon de jeunes a été construit de manière à obtenir des profils différenciés par l'âge, le sexe, le milieu d'origine, le niveau d'études, l'activité. Les candidats à l'entretien ont été rencontrés dans des lieux bien précis : le lycée, l'université, l'espace vert, le souk, le centre-ville, le quartier chic, le quartier populaire, la plage. L'impact des événements de la guerre du Golfe a été mesuré à Casablanca, Fès et Tanger durant les mois de janvier et février 1991. Slogans et anecdotes ont été recueillis. Durant l'automne 1991, un questionnaire comportant des points sur Tailleurs a été soumis à 84 jeunes diplômés inactifs de Salé. En juillet 1993, une série d'entretiens (une douzaine) a eu pour objet d'évaluer l'effet du « temps mondial » sur les représentations du monde au Maroc (voir Bennani-Chraïbi, 1997). Pour ce qui est du versant égyptien, deux visites de deux semaines chacune (décembre 1995, septembre-octobre 1996) et un séjour de deux mois (novembredécembre 1996) nous ont permis d'effectuer plus de vingt-cinq entretiens avec des jeunes Cairotes relativement dissemblables par leur profil, rencontrés à al-Qanâtir (lieu de promenade), aux Pyramides, au Khân al-Khalîlî, à proximité d'une pension pour touristes au centre-ville, etc., et dans les vitrines de l'Occident (Pizza Hut, MacDonald's, Wimpy, Université américaine), dans des quartiers périphériques denses, anciennement informels (Shubra al-Khayma, Imbâba), dans des lieux de résidence des couches moyennes (Madînat Nasr). Des groupes ont été accompagnés dans leurs activités. Par ailleurs, un échantillon diversifié de la presse a été sélectionné en vue d'établir un sondage des différentes positions adoptées autour d'un certain nombre d'événements susceptibles d'être considérés comme des révélateurs des représentations des relations entre l'Occident et le monde musulman (l'attentat d'Oklahoma city, les attentats en Algérie, la Bosnie....).

2. À côté de l'immigration traditionnelle (celle des travailleurs employés essentiellement dans les industries) se développe plus récemment le phénomène de la "nouvelle » immigration. Une étude non publiée analyse ce concept et les enjeux de cette nouvelle donne (voir Belbah, 1990).

3. Sur 1340700 Européens entrés en 1988, 485116 sont français et 330563 espagnol s (Annuaire statistique du Maroc, 1989, p. 244).

4. Le rapport d'Analyse du courrier des téléspectateurs de JV5, établi en mai 1989, confirme cette tendance, en révélant que les deux tiers de la correspondance en provenance des «nouveaux bassins d'audience » (Espagne, Portugal, Grèce, Turquie, Hongrie, Maroc, Algérie) proviennent du Maroc. Les demandes d'adresses de club de correspondances étaient tellement importantes, que TV 5 créa un club en septembre 1989 pour répondre à ces sollicitations.

5. Le français a été introduit pendant le protectorat (1912-1956), notamment par le biais de l'enseignement. En fait, malgré les projets d'arabisation, l'apprentissage et l'emploi de cette 
langue ont connu une grande extension du fait même de l'autre impératif de l'indépendance : la généralisation de l'instruction.

6. L'idéalisation de la partenaire étrangère n'a pas la même signification au Maroc et en Egypte. Dans le premier cas, dans une société gagnée par l'anonymat et l'effondrement subit des repères dits traditionnels, là où la sexualité hors-mariage n'est pas circonscrite à l'espace de la prostitution («l'impur») ou à celui de l'Ailleurs, cela reflète la méfiance à l'égard du groupe d'appartenance. Dans le second cas, l'accès à la partenaire locale en dehors de la relation codifiée du mariage semble rejoindre plus souvent la catégorie de l'impur.

7. Il s'agit d'un périmètre de logements publics qui ont accueilli les réfugiés de Suez pendant le conflit de 1967, ainsi que ceux dont les maisons situées dans la vieille ville ont été détruites, vers la même période, suite à une rude détérioration.

*. (...)

8. D'après l'enquête quantitative que nous avons réalisée au Maroc, durant l'automne 1991, auprès de 84 jeunes des deux sexes, diplômés en quête d'un emploi, ayant entre 24 et 32 ans, et rassemblés en vue d'une action collective, sur les trois cinquièmes des personnes qui ont voulu quitter le Maroc à un moment de leur parcours, les quatre cinquièmes ont effectivement tenté de mettre en œuvre le départ. En dépit du caractère particulier de l'échantillon observé, ces résultats sont révélateurs du poids des « planificateurs » au Maroc.

9. Les résultats de l'enquête quantitative de l'automne 1991 révèlent également que sur les trois cinquièmes qui ont aspiré à un moment ou à un autre à partir, trois cinquièmes considèrent que la vie est difficile « même ailleurs ».

10. Dans l'échantillon égyptien, seuls les interviewés de l'université américaine, généralement issus de familles aisées, et qui ont souvent eu l'occasion de voyager, voire de vivre à l'étranger et donc de se frotter à l'univers occidental, développent un faisceau de stéréotypes plus large: naïveté des Américains, faiblesse de leur culture générale...

11. La migration vers les pays du Golfe suscite souvent les mêmes accusations. Toutefois, ceux qui tiennent ce type de propos, soulignent que c'est Ylnfitâh qui est la source de tous les maux.

12. Ces événements auxquels ont essentiellement participé de jeunes exclus, prêtent à des lectures multiples dans leur polysémie même. Toutefois, la destruction de deux grands hôtels, les Mérinides et le Palais Jamaï, hauts lieux symboliques du tourisme à Fès, fait indéniablement rejaillir la violence sur l'Occident.

13. Au niveau national, le sentiment d'exclusion et le rejet se cristallisent autour de l'État.

\section{INDEX}

Mots-clés : identité, jeunesse, Maroc, Occident 